\title{
Analyse des besoins en équipements et matériels de production des spéculations des pôles de développement agricole du Bénin
}

\author{
Emile C. AGBANGBA ${ }^{1 *}$, Patrice ADEGBOLA ${ }^{2}$, Gustave D. DAGBENONBAKIN ${ }^{1}$, \\ Paul HOUSSOU ${ }^{3}$, Fortuné OGOUVIDE ${ }^{1}$ et Hounyevou Klotoe AGOSSOU ${ }^{3}$
${ }^{l}$ Institut National des Recherches Agricoles du Bénin, Programme d'Amélioration de la Productivité Agricole des Petits Exploitants (PAPAPE).
${ }^{2}$ Institut National des Recherches Agricoles du Bénin, Centre de Recherche Agricole d'Agonkanmey.
${ }^{3}$ Institut National des Recherches Agricoles du Bénin, Programme de Technologie Agricole et Alimentaire. \\ "Auteur correspondant ; E-mail : agbangbacodjoemile@gmail.com
}

\section{RESUME}

L'agriculture béninoise est caractérisée par une faible utilisation d'intrants et l'emploi de matériels et équipements pré et post récolte rudimentaires. Cette étude visait à développer une approche participative pour identifier les matériels et équipements agricoles répondant aux besoins des producteurs du Bénin. Des villages ont été choisis dans six Pôles de Développement Agricoles du Bénin (PDA). Dans chaque village, une assemblée villageoise d'effectif variant entre 26 et 60 producteurs choisis de façon aléatoire et des focus groupes ont été organisés pour identifier les principales cultures contribuant au revenu et à la sécurité alimentaire. De plus, les besoins en équipements/matériels selon chaque contrainte majeure de production ont été identifiés par filière. Une régression logistique polytomique ordinale a été réalisée pour examiner si les rangs attribués varient en fonction des spéculations et/ou des villages ou Pôle de Développement Agricole. Une classification automatique de données de rang multivariées incomplètes a été utilisée pour évaluer les rangs des différentes opérations qui constituent des goulots d'étranglement. Les résultats révèlent qu'il y une variation significative $(\mathrm{p}<0,001)$ des rangs attribués aux cultures contribuant à l'alimentation et au revenu suivant les villages, les spéculations et les PDA et quelle que soit la spéculation concernée, le labour et le sarclage apparaissent comme les deux premières opérations les plus cités et qui constituent des goulots d'étranglement pour les producteurs. Des matériels et équipements de production ont été proposés de façon participative aux producteurs pour lever les contraintes.

(C) 2018 International Formulae Group. All rights reserved.

Mots clés: Mécanisation, pré-récolte, revenu, sécurité alimentaire.

\section{Analysis of agricultural machinery and equipment requirements for Benin's Agricultural Development Poles}

\begin{abstract}
Beninese agriculture is characterized by low input and the use of rudimentary pre- and post-harvest materials and equipment. This study aimed to develop a participatory approach to identify agricultural materials and equipment that meet the needs of producers in Benin. Villages were selected in six Benin Agricultural Development Poles. In each village, an assembly of 26 to 60 randomly selected producers and focus groups were organized to identify the main crops contributing to income and food security. In addition,
\end{abstract}


the equipment / material requirements according to each major production constraint were identified by sector. An ordinal polytomic logistic regression was carried out to examine if the ranks assigned vary according to the speculations and / or the villages or Agricultural Development Pole. An automatic classification of incomplete multivariate rank data was used to evaluate the ranks of the various operations that constitute bottlenecks. The results revealed that there was a significant variation $(p<0.001)$ in the ranks attributed to the crops contributing to food and income according to the villages, speculations and Agricultural Development Poles and whatever the speculation concerned, plowing and weeding appear as the first two most cited operations and were bottlenecks for producers. Production materials and equipment have been proposed in a participatory manner to producers to overcome constraints.

(C) 2018 International Formulae Group. All rights reserved.

Keywords: Mechanization, pre-harvest, income, food security.

\section{INTRODUCTION}

En Afrique de l'Ouest, la force de travail dépend dans une écrasante majorité de la force physique avec l'utilisation d'outils manuels comme la houe pour exécuter les différentes opérations culturales (Yuan, 2016). Ces outils ont des limites implicites en termes d'énergie et de rendement potentiel, en particulier dans un environnement tropical. Ainsi, le secteur agricole est caractérisé par une faible productivité (Ahouandjinou et al., 2010). Cela s'explique par le fait que l'agriculture ne bénéficie pas encore des techniques et méthodes modernes pour son développement tant au niveau de la production que de la conservation, de la transformation et de la commercialisation des produits agricoles. L'investissement dans l'équipement agricole a permis aux agriculteurs d'intensifier la production, d'améliorer leurs revenus et leur qualité de vie dans des pays comme l'Inde, la Chine, le Brésil et la Turquie (Yuan, 2016). Ces pays sont actuellement de grands producteurs et des leaders mondiaux en exportation de machines agricoles. La même situation pourrait se produire en Afrique si les agriculteurs pouvaient intensifier leurs activités grâce à une plus grande mécanisation. La mécanisation agricole revêt une importance surtout pour la préparation du sol (Vall et al., 2006). En effet, il est très difficile d'imaginer actuellement un secteur agricole se développer sans une technologie améliorée (Atidegla et al., 2017). La mécanisation agricole destinée à la multitude de petits exploitants agricoles en Afrique subsaharienne (ASS) a été une question négligée depuis trop longtemps (FAO, 2016).
Les différents programmes de mécanisation de l'agriculture mis en œuvre dans les pays d'Afrique Subsaharienne entre 1960 et la fin des années 1990 à travers le soutien au développement de la mécanisation attelée et de la mécanisation motorisée ont obtenu des résultats en dessous des attentes (Clarke et Bishop, 2002). Pourtant, l'un des facteurs clés de la croissance du secteur de l'agriculture en Asie (révolution verte) et en Amérique latine a été la mécanisation.

$\mathrm{Au}$ Bénin, plusieurs localités ont bénéficié d'appui des structures en matière d'équipements/matériels agricoles (Atidegla et al., 2017; Ahouandjinou et al., 2010). Lesdites structures sont de types variables: projets, programmes, ONG, gouvernement. La majorité des interventions ont connu un succès mitigé comme la plupart des pays subsahariens (Balse et al., 2015). Les raisons de l'échec de l'introduction de la mécanisation dans l'agriculture inclurent l'inadéquation des équipements à des besoins des agriculteurs, le manque de pièces de rechange et d'entretien (Fonteh, 2010) conduisant à l'incapacité d'entretenir et de renouveler les équipements. Des résultats récents suggèrent que l'analyse des perspectives de la mécanisation doit prendre en compte les changements socioéconomiques en cours, principalement l'explosion démographique, l'urbanisation croissante, l'augmentation continue des prix des produits pétroliers, les besoins des agriculteurs, les orientations politiques des gouvernements (Side et Havard, 2015).

Ainsi, pour une mécanisation agricole durable, les besoins réels des producteurs en équipement agricole méritent d'être identifiés. Ceci passe par le développement d'une 
méthodologie pour identifier les besoins réels des producteurs. Cette étude visait à développer une approche participative pour identifier les matériels et équipements agricoles répondant aux besoins des producteurs.

\section{MATERIEL ET METHODES \\ Zones d'étude}

L'étude a été menée dans 18 villages sur 30 du Programme d'Amélioration de la Productivité Agricole des Petits Exploitants (PAPAPE) répartis dans six Pôles de Développement Agricole (PDA) du Bénin (Tableau 1, Figure 1). Le choix de ces villages a été fait en tenant compte de la zone de production, de la présence et du niveau de production des spéculations agricoles.

\section{Méthodes de collecte des données}

Une assemblée villageoise d'effectif variant entre 26 et 60 producteurs/trices choisis de façon aléatoire a été organisée dans chaque village. Des interviews semi structurées ont été réalisées à l'aide du guide d'entretien préalablement conçu. Une partie du guide a été administré en plénière avec l'ensemble des participants. Cette étape a permis d'identifier, entre autres, les principales cultures contribuant au revenu et à la sécurité alimentaire. Par ailleurs, de manière participative, il a été retenu avec l'ensemble des acteurs les trois principales cultures vivrières contribuant le plus au revenu et à la sécurité alimentaire des villageois.

Les participants ont été ensuite répartis en trois sous-groupes selon les trois principales cultures vivrières de leur village. Chaque sous-groupe est constitué par des producteurs/productrices de toutes les tranches d'âge (vieux, jeunes et adultes). Cette étape a permis de faire le point des équipements/matériels utilisés pour toutes les opérations culturales ainsi que les différentes contraintes rencontrées. Par ailleurs, les différentes caractéristiques des équipements déjà utilisés ainsi que les améliorations souhaitées ont été identifiées. De plus, les besoins en équipements/matériels selon chaque contrainte majeure de production ont été identifiés par filière.

Les participants au focus groupe sont revenus en plénière afin de faire le point des équipements identifiés par filière et les valider. Cette étape a permis d'exposer les équipements déjà existants et de vérifier ainsi leurs caractéristiques afin de permettre aux participants de choisir en fonction de leurs besoins, de leurs capacités d'utilisation et de production.

\section{Méthodes d'analyse}

Des histogrammes ont été tracés traduisant la fréquence de citation des spéculations dans les villages parcourus. Une régression logistique polytomique ordinale a été réalisée pour examiner si les rangs attribués varient en fonction des spéculations et/ou des villages ou Pôle de Développement Agricole. Le package ordinal (Christensen, 2015) du logiciel R-3.4.0 (R Core Team, 2017) avec la fonction $c l m$ et le lien logit a été utilisé. Dans le cas où un facteur (spéculation ou village ou pôle de développement) est significatif, les six premières spéculations les plus citées ont été considérées pour voir comment leur rang de classement varie d'un village à un autre et d'une spéculation à l'autre. Des diagrammes en toile d'araignée ont été alors réalisés à cet effet avec la fonction Radarchart.

Les fréquences de citation des opérations de pré-récolte qui constituent des goulots d'étranglement pour chaque spéculation dans les villages ont été représentées par des histogrammes. Une classification automatique de données de rang multivariées incomplètes (Grimonprez et Jacques, 2016) a été utilisée pour évaluer les rangs des différentes opérations qui constituent des goulots d'étranglement avec la fonction rankcluster implémentée dans le package Rankcluster du logiciel R -3.4.0 (R Core Team, 2017).

Enfin ces différents résultats ont permis d'identifier les besoins des producteurs et transformateurs en matériels et équipements agricoles. 
Tableau 1: Répartition des villages par Pôle de Développement Agricole (PDA) et par zone agroécologique (ZAE), communes et villages de la zone d'étude.

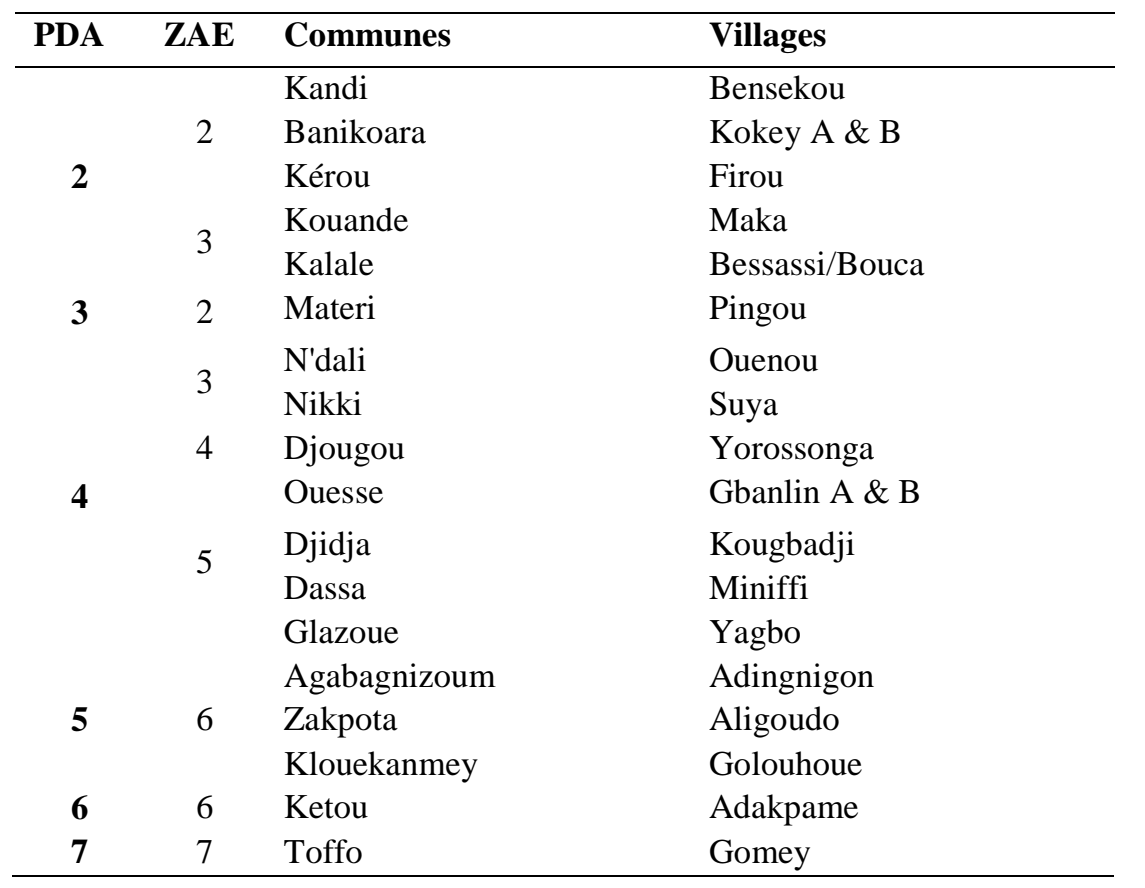

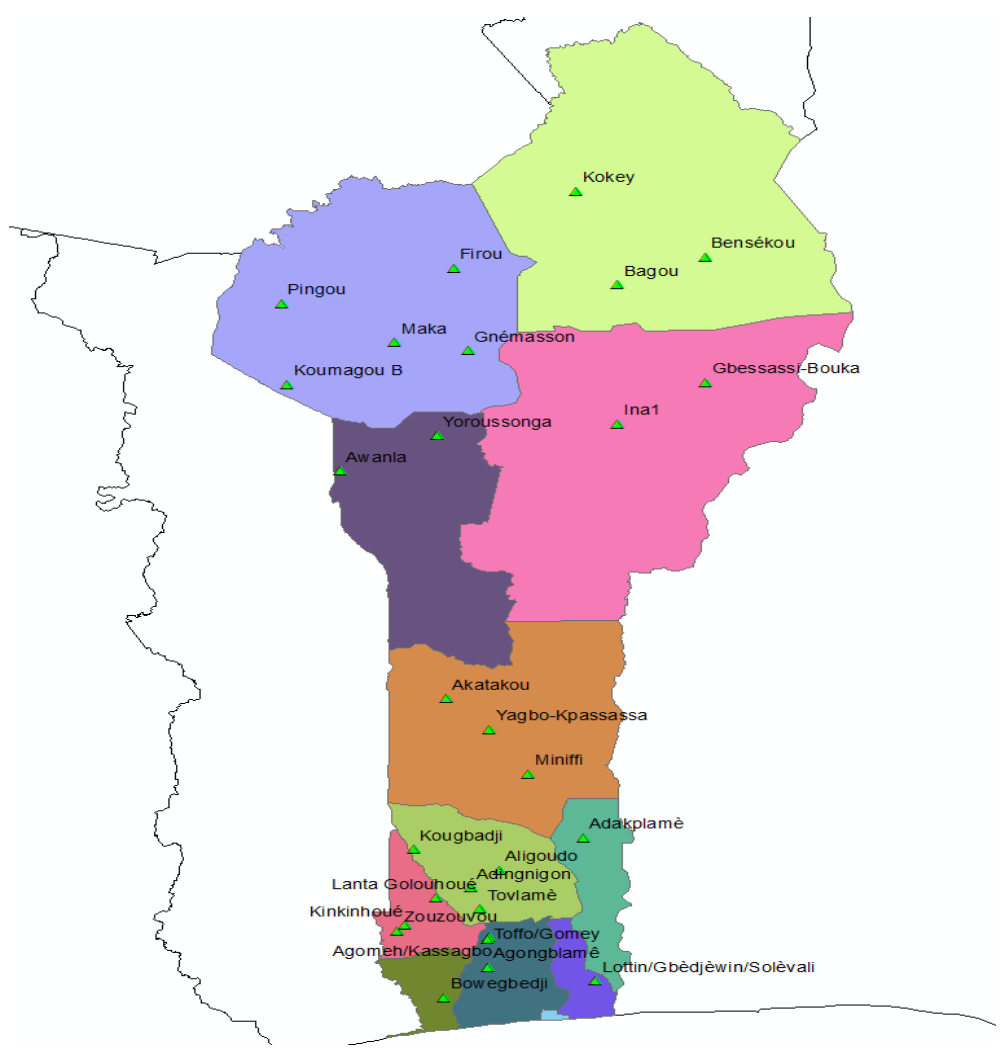

Figure 1 : Villages d'intervention du Programme d'Amélioration de la Productivité Agricole des Petits Exploitants (PAPAPE). 
RESULTATS

\section{Importance des cultures selon leur apport au revenu et à l'alimentation}

De l'analyse de la Figure 2 (a), il ressort que c'est le soja qui est la principale spéculation la plus citée comme source de revenus. En plus de cette spéculation, le maïs, le niébé, l'arachide et le manioc sont les cultures vivrières les plus évoquées rapportant plus de revenus aux populations des zones d'enquêtes. Le riz, une des cultures phares du Programme d'Amélioration de la Productivité des Petites Exploitations (PAPAPE), est moyennement citée comme spéculation source de revenu. Contrairement à ces spéculations, ce sont les spéculations comme le petit mil, la patate douce ou le dohicoun qui sont, entre autres, les spéculations les moins citées comme source de revenus.

Les spéculations comme le mais, le niébé et le manioc sont les principales spéculations évoquées contribuant à la sécurité alimentaire (Figure 2 b). L'igname, l'arachide et le riz sont également des spéculations citées qui contribuent moyennement à la sécurité alimentaire des populations. La patate douce, l'oranger et le gombo sont les spéculations les moins évoquées contribuant à la sécurité alimentaire.

En somme, il ressort de la Figure 2 (a) et (b) que ce sont les spéculations comme le maïs, le niébé et le soja qui contribuent à la fois à la sécurité alimentaire et au revenu.

\section{Variation des rangs attribués aux cultures suivant les villages et les PDA}

Afin de voir s'il y a une variation des rangs attribués aux spéculations suivant les villages et les PDA, et une variation des rangs en fonction des spéculations, une régression logistique multiple a été faite sur la classification des spéculations pour leur apport au revenu et à la sécurité alimentaire (Tableau 2). Les probabilités associées à la spéculation et aux villages suivant la classification des cultures par rapport au revenu sont significatives au seuil de $1 \%$. On en déduit donc qu'il y a une variation des rangs attribués aux cultures suivant les villages. De même, les probabilités associées aux PDA et aux spéculations sont toutes significatives au seuil de $1 \%$. On en déduit aussi qu'il a une variation des rangs attribués aux cultures suivant les PDA.

Pour la classification des cultures par rapport à la sécurité alimentaire, les probabilités associées aux spéculations, aux villages et aux PDA sont toutes significatives au seuil de $1 \%$. Il ressort donc qu'il y a une variation des rangs attribués aux cultures contribuant à l'alimentation suivant les villages, les spéculations et les PDA.

\section{Répartition des cultures par village selon leur apport au revenu et à l'alimentation}

Les Figures 3 (a) et (b) montrent la répartition des spéculations dans les villages suivant l'apport au revenu et à la contribution à la sécurité alimentaire. De la Figure 3 (a), il ressort que les spéculations qui apparaissent le plus au premier rang comme contributeur au revenu sont le maïs dans 10 des 18 villages (Adakplamey, Adingnigon, Bensekou, Bessassi-Bouka, Firou, Gbanlin, Gomey, Kokey, Kougbadji, Maka et Kougbadji), le soja dans 3 villages (Yagbo, Ouenou et Miniffi), l'arachide dans 2 villages (Aligoudo et Yorossonga), le manioc dans 2 villages (Suya et Golouhoue) et le niébé dans 1 village (Pingo).

La Figure 3 (b), par ailleurs, montre clairement que c'est le maïs qui apparait au premier rang dans tous les 18 villages comme l'unique spéculation contribuant à la sécurité alimentaire des populations. Cette spéculation est suivie du manioc qui se trouve être la culture contribuant au second rang à la sécurité alimentaire des populations de 10 des 18 villages (Adakplamey, Yorossonga, Bessassi, Gbanlin, Golouhoue, Gomey, Kougbadji, Maka, Minifi, et Ouenou). En plus du manioc, le niébé dans 4 villages (Aligoudo, Adingnigon, Firou et Pingou), le soja dans 2 villages (Suya et Bensekou), et le riz dans 2 villages (Yagbo et Kokey) contribuent au second rang à la sécurité alimentaire des populations.

Les spéculations qui contribuent plus à la fois au revenu et à la sécurité alimentaire des populations sont le maïs, le manioc et le soja. 


\section{Répartition des cultures par pôle selon leur apport au revenu et à l'alimentation}

Les Figures 4 (a) et (b) montrent l'importance des cultures suivant les PDA. Il ressort que le maïs est au premier rang la spéculation contribuant au revenu des populations dans la majorité des $\operatorname{PDA}(2,7,6$ et 5). Toujours au premier rang, le soja et le niébé sont les spéculations qui contribuent plus au revenu, respectivement dans les PDA 3 et 4.

La Figure 4 (b) montre tout comme la Figure 3 (b) que c'est le maïs qui est au premier rang des spéculations contribuant à la sécurité alimentaire des populations des tous les PDA considérés pour cette étude (pôles 2, $3,4,5,6$ et 7). Viennent au second rang le manioc dans 4 pôles $(7,6,5$ et 4$)$, le niébé dans 2 pôles (5 et 3 ) et le riz dans un pôle (2).

Il apparait que c'est le maïs qui contribue à la fois à la sécurité alimentaire et au revenu des populations dans la grande majorité des PDA (pôles 2, 7, 6, 5 et 4).

\section{Caractéristiques des équipements/matériels utilisés dans les opérations pré-récolte dans les villages et par spéculation}

Dix (10) différents types de matériels de pré-récolte ont été recensés dans les villages. Il s'agit de : tracteur à 2 roues motrices de $57 \mathrm{CV}$, tracteur à 2 roues motrices de $30 \mathrm{CV}$, tracteur à 4 roues motrices de 75 $\mathrm{CV}$, charrue à disques, remorque de tracteur, remorque de charrette, pulvérisateur à dos, pulvérisateur à pile et pulvérisateur à moteur (Tableau 3).

Classement des opérations culturales selon leur niveau de difficulté de réalisation et besoins en équipements

Quelle que soit la spéculation concernée, le labour et le sarclage apparaissent comme les deux premières opérations les plus citées et qui constituent des goulots d'étranglement pour les producteurs (Figure 5). Par ailleurs, pour le maïs, le soja, le niébé et l'arachide, le semis est également l'une des opérations les plus citées. L'opération la moins citée est la pulvérisation ou le traitement phyto sanitaire des plantes. Les résultats du classement des contraintes ont révélé que globalement, le labour, la préparation du sol, le sarclage et le semis sont les opérations les plus contraignantes (Tableau 4). Les équipements identifiés par spéculation avec les producteurs sont consignés dans le Tableau 5.

De l'analyse de ce tableau, il ressort que les besoins des producteurs en matériels et équipements agricoles concernent les outils de travail du sol (essoucheuses, tracteurs et motoculteurs avec accessoires), les outils de pulvérisation (pulvérisateur manuel ou motorisé), les sarcleuses et récolteuses motorisées. Le semis doit être réalisé à l'aide de semoir en ligne.
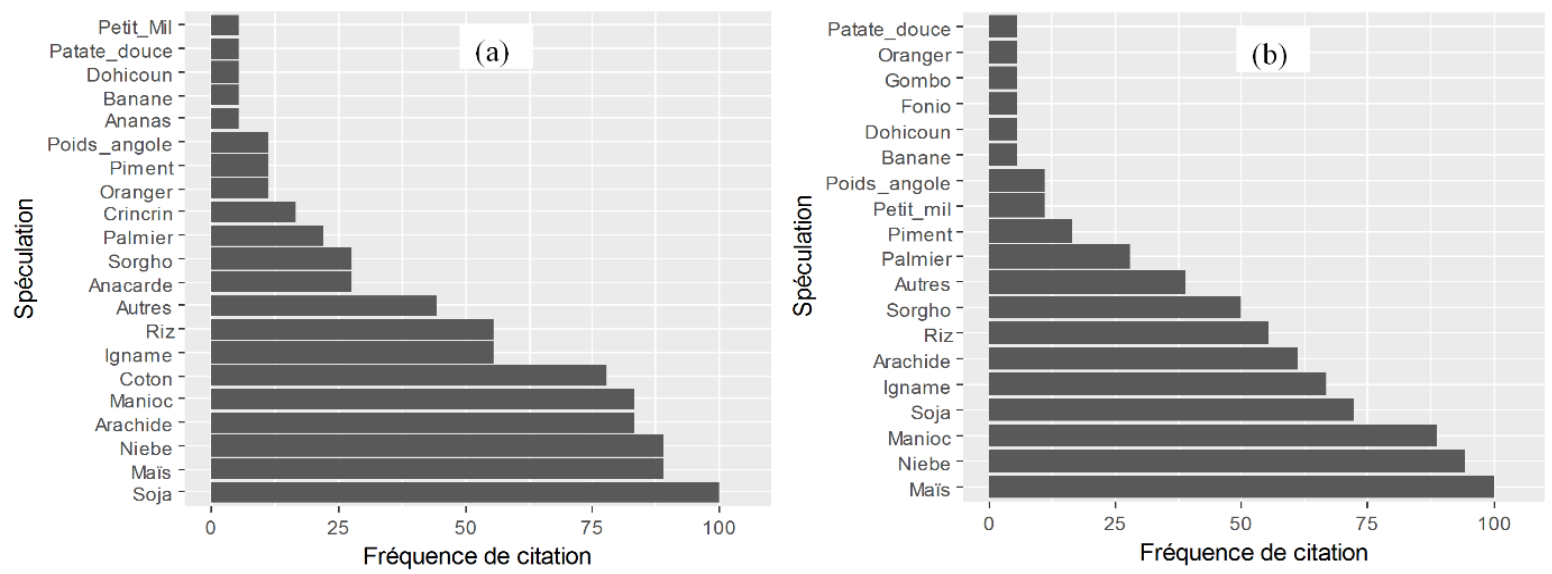

Figure 2: Fréquence de citation des spéculations selon leur apport au revenu (a) et à la sécurité alimentaire (b) par village. 
Tableau 2: Résultats du CLM sur la classification des spéculations pour leur apport au revenu et à la sécurité alimentaire dans les villages et PDA.

\begin{tabular}{lllllll}
\hline Facteurs & \multicolumn{2}{l}{$\begin{array}{l}\text { Classification des cultures par } \\
\text { rapport au revenu }\end{array}$} & \multicolumn{3}{l}{$\begin{array}{l}\text { Classification des cultures par } \\
\text { rapport à la sécurité alimentaire }\end{array}$} \\
\hline Spéculation & Df & Chisq & Prob. & Df & Chisq & Prob. \\
Village & 17 & 144.402 & $<0.001$ & 9 & 50.69 & $<0.001$ \\
Pole & 5 & 40.42 & 0.001 & 17 & 90.29 & $<0.001$ \\
Spéculation & 20 & 94.65 & $<0.001$ & 5 & 63.17 & $<0.001$ \\
\hline
\end{tabular}

(a)

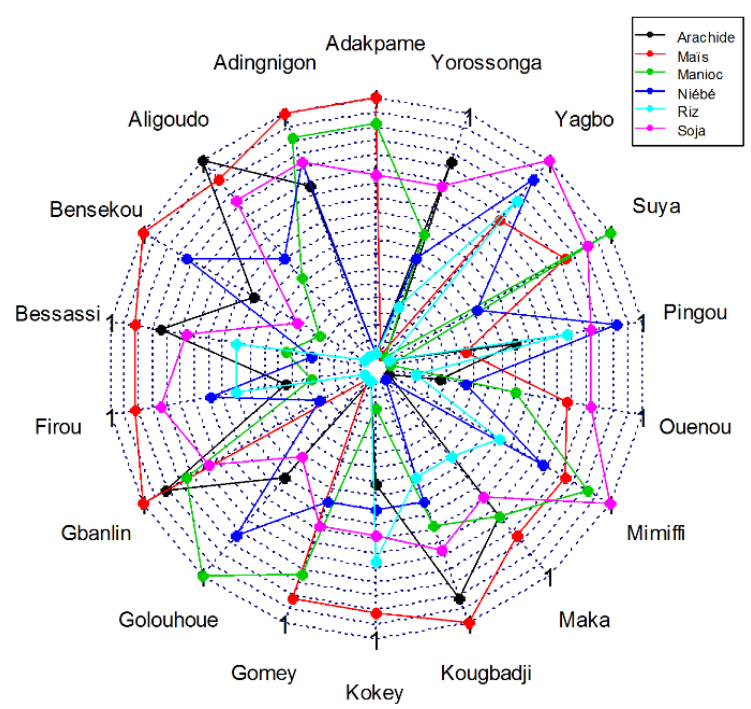

(b)

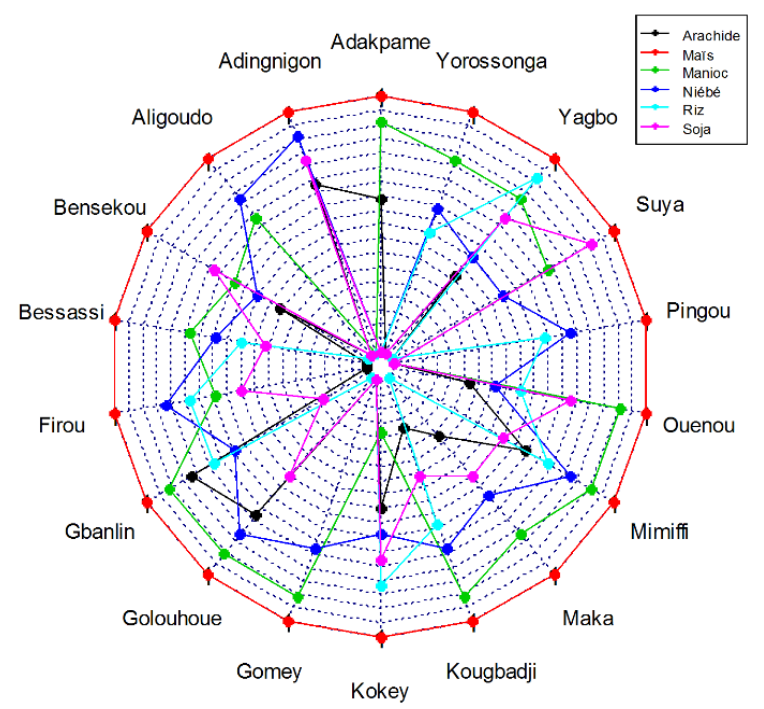

Figure 3: Diagramme en toile d'araignée montrant la classification des spéculations suivant l'apport au revenu (a) et à la sécurité alimentaire (b).

(a)

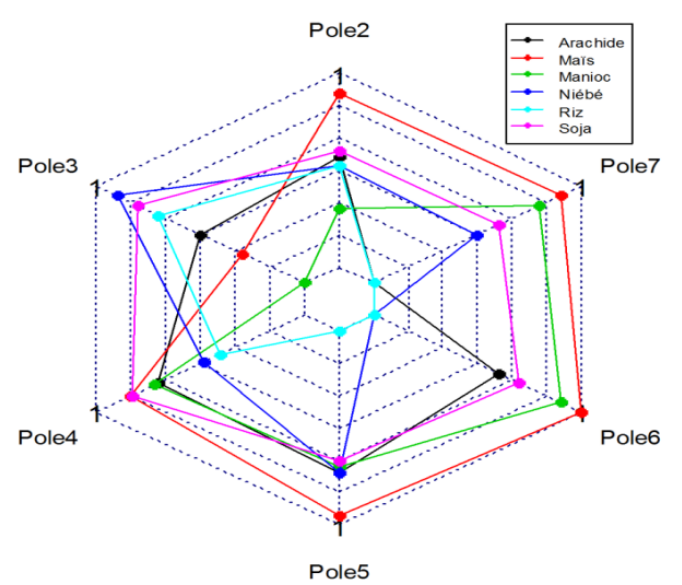

(b)

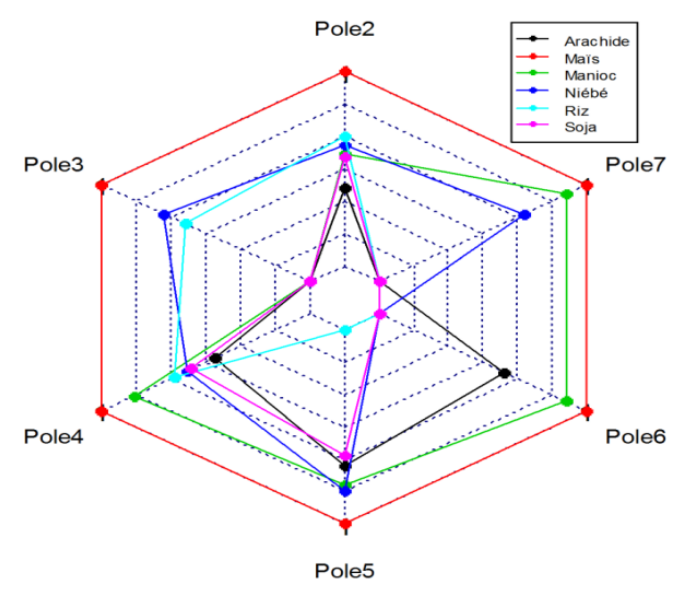

Figure 4: Diagramme en toile d'araignée montrant la classification des spéculations par apport au revenu (a) et à la sécurité alimentaire (b) suivant les pôles de développement. 
Tableau 3: Equipements pré-récolte recensés dans les pôles de développement.

\begin{tabular}{|c|c|c|c|c|c|c|}
\hline $\begin{array}{l}\text { Pôle de } \\
\text { développement } \\
\text { Agricole (PDA) }\end{array}$ & Spéculations & $\begin{array}{l}\text { Désignation } \\
\text { du matériel }\end{array}$ & Marque & $\begin{array}{l}\text { Opérations } \\
\text { culturales }\end{array}$ & $\begin{array}{l}\text { Caractéristiques } \\
\text { du matériel }\end{array}$ & $\begin{array}{l}\text { Etat } \\
\text { actuel du } \\
\text { matériel }\end{array}$ \\
\hline \multirow{5}{*}{2} & Toutes & Tracteur & $\begin{array}{l}\text { Mahindra } \\
\text { type } 605 \mathrm{D} 1\end{array}$ & Traction & $\begin{array}{l}2 \text { roues motrices } \\
\text { de } 57 \mathrm{CV}\end{array}$ & Neuf \\
\hline & Toutes & $\begin{array}{l}\text { Charrue à } \\
\text { disques }\end{array}$ & $\begin{array}{l}\text { Pour } \\
\text { tracteur de } \\
60 \mathrm{CV}\end{array}$ & Labour & 3 disques & Bon \\
\hline & Toutes & Tracteur & Chinoise & Labour & $\begin{array}{l}4 \text { roues motrices } \\
\text { de } 75 \mathrm{CV}\end{array}$ & En panne \\
\hline & Toutes & $\begin{array}{l}\text { Charrue à } \\
\text { disques }\end{array}$ & $\begin{array}{l}\text { Pour } \\
\text { tracteur de } \\
55-60 \mathrm{CV}\end{array}$ & Labour & 3 disques & Bon \\
\hline & Toutes & $\begin{array}{l}\text { Charrue à } \\
\text { disque }\end{array}$ & $\begin{array}{l}\text { Pour } \\
\text { tracteur de } \\
55-60 \mathrm{CV}\end{array}$ & Labour & 3 disques & Bon \\
\hline 3 & Toutes & $\begin{array}{l}\text { Charrette } \\
\text { animale }\end{array}$ & COBEMAG & Transport & 500 à $1000 \mathrm{Kg}$ & Bon \\
\hline \multirow{2}{*}{4} & $\begin{array}{l}\text { Maïs, Niébé, } \\
\text { Soja }\end{array}$ & $\begin{array}{l}\text { Pulvérisateur } \\
\text { à pile }\end{array}$ & & $\begin{array}{l}\text { Traitement } \\
\text { chimique }\end{array}$ & $\begin{array}{l}\text { Capacité du } \\
\text { réservoir 1,5 } \\
\text { litres }\end{array}$ & Bon \\
\hline & $\begin{array}{l}\text { Maïs, Niébé, } \\
\text { Soja }\end{array}$ & $\begin{array}{l}\text { Pulvérisateur } \\
\text { à dos }\end{array}$ & & $\begin{array}{l}\text { Traitement } \\
\text { chimique }\end{array}$ & $\begin{array}{l}\text { Capacité du } \\
\text { réservoir 15-18 } \\
\text { litres }\end{array}$ & Bon \\
\hline 5 & $\begin{array}{l}\text { Maïs, Niébé, } \\
\text { Soja }\end{array}$ & $\begin{array}{l}\text { Pulvérisateur } \\
\text { à dos }\end{array}$ & $\begin{array}{l}\text { Marque } \\
\text { RAINBOW }\end{array}$ & $\begin{array}{l}\text { Traitement } \\
\text { chimique }\end{array}$ & $\begin{array}{l}\text { Capacité du } \\
\text { réservoir 15-18 } \\
\text { litres }\end{array}$ & Bon \\
\hline \multirow{2}{*}{6} & $\begin{array}{l}\text { Maïs, Niébé, } \\
\text { Soja }\end{array}$ & $\begin{array}{l}\text { Pulvérisateur } \\
\text { à dos }\end{array}$ & $\begin{array}{l}\text { Marque } \\
\text { AYEKOO }\end{array}$ & $\begin{array}{l}\text { Traitement } \\
\text { chimique }\end{array}$ & $\begin{array}{l}\text { Capacité du } \\
\text { réservoir 15-18 } \\
\text { litres }\end{array}$ & Bon \\
\hline & $\begin{array}{l}\text { Maïs, Niébé, } \\
\text { Soja }\end{array}$ & $\begin{array}{l}\text { Pulvérisateur } \\
\text { à pile }\end{array}$ & & $\begin{array}{l}\text { Traitement } \\
\text { chimique }\end{array}$ & $\begin{array}{l}\text { Capacité du } \\
\text { réservoir 1,5 } \\
\text { litres }\end{array}$ & Bon \\
\hline \multirow{3}{*}{4} & Toutes & Tracteur & Chinoise & Labour & $\begin{array}{l}2 \text { roues motrices } \\
\text { de } 30 \mathrm{CV}\end{array}$ & En panne \\
\hline & $\begin{array}{l}\text { Maïs, Niébé, } \\
\text { Soja }\end{array}$ & $\begin{array}{l}\text { Pulvérisateur } \\
\text { à dos }\end{array}$ & $\begin{array}{l}\text { Marque } \\
\text { TOPAZ }\end{array}$ & $\begin{array}{l}\text { Traitement } \\
\text { chimique }\end{array}$ & $\begin{array}{l}\text { Capacité du } \\
\text { réservoir 15-18 } \\
\text { litres }\end{array}$ & Bon \\
\hline & $\begin{array}{l}\text { Maïs, Niébé, } \\
\text { Soja }\end{array}$ & $\begin{array}{l}\text { Pulvérisateur } \\
\text { à moteur }\end{array}$ & Ghanéenne & $\begin{array}{l}\text { Traitement } \\
\text { chimique }\end{array}$ & $1,5 \mathrm{CV}$ & Bon \\
\hline
\end{tabular}



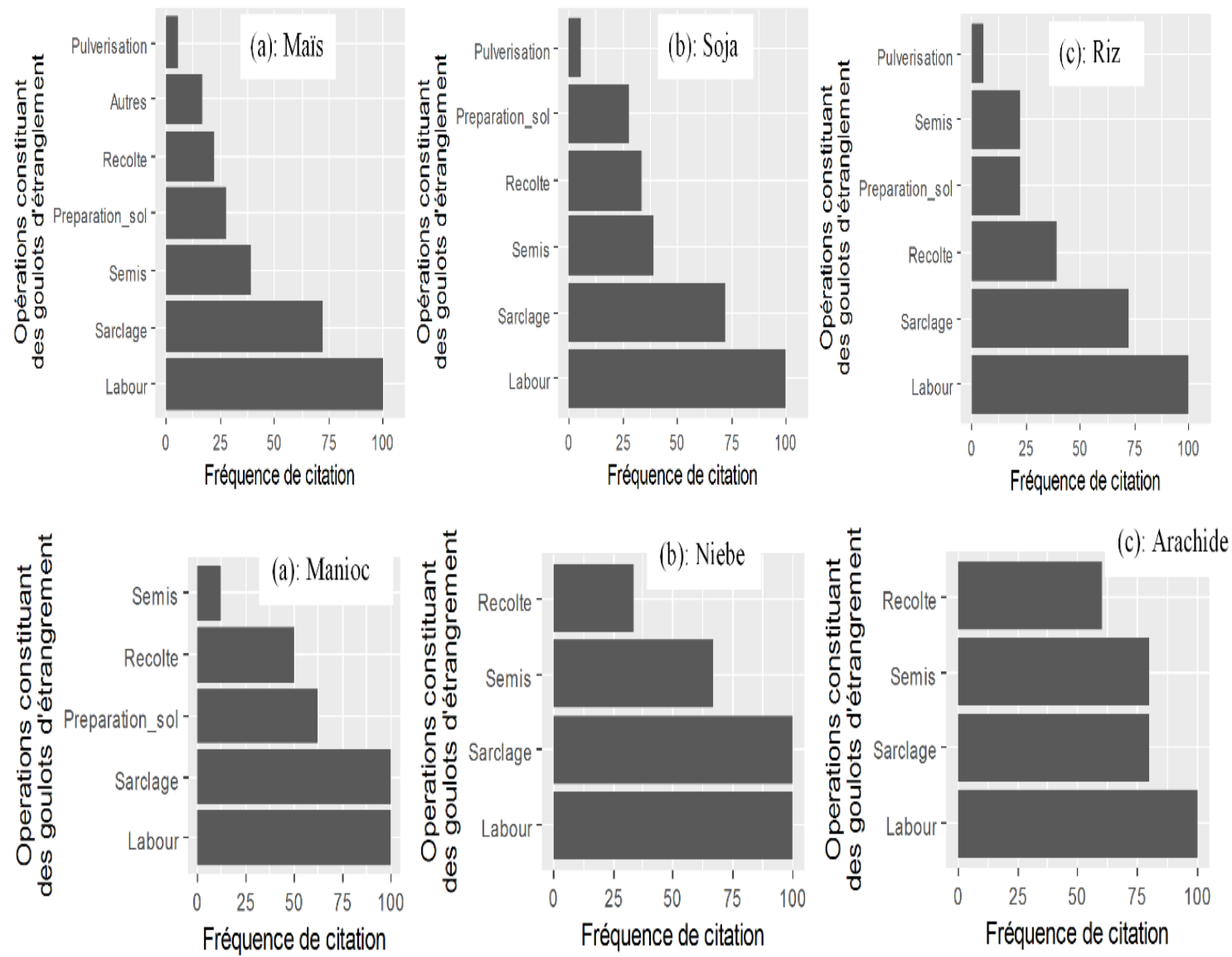

Figure 5: Opérations pré-récolte constituants des goulots d'étranglement.

Tableau 4: Résultats du Rank cluster sur le classement sur les opérations qui constituent des goulots d'étranglement.

\begin{tabular}{lccccccc}
\hline Cultures & $\begin{array}{c}\text { Préparation } \\
\text { du Sol }\end{array}$ & Labour & Sarclage & Semis & Pulvérisation & Récolte & $\boldsymbol{\pi}$ \\
\hline Maïs & 6 & 1 & 2 & 3 & 4 & 5 & 0,79 \\
Manioc & 1 & 2 & 3 & 4 & 5 & - & 0,74 \\
Niébé & - & 1 & 2 & 3 & - & 4 & 0,69 \\
Arachide & - & 1 & 2 & 3 & - & 4 & 0,85 \\
Soja & 1 & 2 & 3 & 4 & - & 5 & 0,74 \\
Riz & - & 1 & 2 & 3 & - & 4 & 0,63 \\
\hline
\end{tabular}


Tableau 5: Synthèse des matériels et équipements de production retenus avec les producteurs par spéculation.

\begin{tabular}{lll}
\hline Maïs & Soja & Manioc \\
\hline Essoucheuse motorisée & Essoucheuse motorisée & Essoucheuse motorisée \\
Motoculteur/tracteur avec & Motoculteur/tracteur avec & Motoculteur/tracteur avec \\
accessoires & accessoires & accessoires \\
Semoir & Semoir en ligne & Sarcleuse motorisée \\
Pulvérisateur à pile/battérie & $\begin{array}{l}\text { Pulvérisateur } \\
\text { manuelle/motorisée }\end{array}$ & Pulvérisateur à pile/battérie \\
Kit d'habillement & Bineuse & Bineuse \\
Sarcleuse motorise & Sarcleuse motorise & Récolteuse motorisée \\
Bineuse & Bineuse & Remorque de tracteur ou de \\
& & motoculteur \\
Remorque & Récolteuse motorisée & \\
\hline Arachide & Riz & Niébé \\
\hline $\begin{array}{l}\text { Motoculteur/tracteur avec } \\
\text { accessoires }\end{array}$ & Motoculteur/tracteur avec & Tracteurs et accessoires \\
Semoir & accessoires & \\
Bineuse & Pulvérisateur & Semoir en ligne \\
Bineuse tractée & Semoir en ligne & Pulvérisateur motorisée \\
Récolteuse motorisée & Moissonneuse batteuse & Sarcleuse motorise \\
\hline
\end{tabular}

\section{DISCUSSION}

Les principales spéculations qui participent à la sécurité alimentaire et au revenu des ménages sont le maïs, le soja, niébé, arachide, manioc et le riz. Plusieurs auteurs ont déjà montré l'importance de ces cultures dans le régime alimentaire et le revenu des producteurs. Le maïs constitue la principale céréale intervenant dans l'alimentation humaine, l'alimentation animale et dans l'amélioration du revenu des producteurs (Soulé et al., 2008; Jaliya et al., 2008). Le niébé représente une source substantielle de protéines végétales et contribue dans la sécurité alimentaire pour répondre à la demande alimentaire et la réduction de la pauvreté (Allogni et al., 2004). Le soja aussi participe à la sécurité alimentaire par son apport important en protéine et en huile végétale pour les populations à faible revenu (Appunu et al., 2008; Li-juan et al., 2010). Par ailleurs, bien que le riz ne soit pas la première culture de choix des producteurs, les besoins en riz sont croissants en Afrique sub-saharienne, en raison de la poussée démographique qui est de $4 \%$ par an et de l'intérêt accordé au riz par les populations locales (Balasubramanian et al., 2007; Adégbola et al., 2002). L'augmentation sans cesse croissante des besoins alimentaires des ménages, suite à la pression démographique entraîne le plus souvent le changement d'objectifs au niveau des populations. Dans cette perspective, l'une des stratégies dont font souvent recours les producteurs est l'augmentation de la superficie cultivable. Cependant l'agriculture reste peu développée au Bénin (Yuan, 2016) et la productivité est statique. Emblaver toutes les superficies à temps afin de tirer profit des conditions climatiques de plus en plus instables devient une source de réflexion pour les producteurs.

Le labour, le planage, le buttage et le sarclo-buttage constituent des opérations phares de la phase de pré-production et s'avèrent trop contraignantes pour la grande majorité des producteurs des villages d'étude. Cette pénibilité est principalement due à l'utilisation des outils rudimentaires et à la présence dans les nouvelles terres cultivables d'arbres et de souches d'arbre ; ce qui réduit les superficies emblavées. Or la mécanisation diminue la pénibilité du travail et améliore potentiellement l'attractivité du secteur 
agricole, en particulier pour les jeunes (Vall et al., 2006 ; Balse et al., 2015). Pourtant sur les 18 villages d'étude, seuls trois tracteurs sont retrouvés dont un seul en bon état. Ce constat est le même dans la plupart des pays subsahariens où la motorisation de la production agricole a stagné car la majorité des programmes d'Etat et projets pour le développement de la mécanisation a disparu, et peu de privés ont pris le relais (Side et Havard, 2015). Ainsi, entre 1980 et 2003, le nombre de tracteurs en utilisation pour 1000 ha de terres arables est passé de 2 à 1,3 (Mrema et al., 2008). Les principaux facteurs qui influent la mécanisation agricole sont l'intensification de l'agriculture, les compétences des utilisateurs (Bordet, 1997), la croissance des capitaux disponibles et l'amélioration des routes (Binswanger et Donovan, 1987) et l'adéquation des équipements et les besoins des utilisateurs (Side et Havard, 2015). Par conséquent, les mesures d'accompagnement du processus de mécanisation agricole doivent être mises en place en étroite collaboration entre les gouvernements, les producteurs, les privés, les structures de recherche et de formation. Cette nouvelle approche de mécanisation, sur la base d'une demande construite, doit permettre aux agriculteurs d'interagir efficacement avec les fournisseurs de services de mécanisation agricole en fonction de leurs besoins et des projets de développement clairs et précis de leurs exploitations agricoles.

\section{Conclusion}

Cette étude visait à développer une approche participative pour identifier les matériels et équipements agricoles répondant aux besoins des producteurs. Elle a permis d'identifier les cultures les plus importantes pour leur contribution au revenu et à la sécurité alimentaire dans les PDA du Bénin. Les opérations culturales les plus pénibles aux producteurs sont le labour, la préparation du sol, le sarclage et le semis pour lesquelles des matériels et équipements agricoles sont proposés de façon participative pour lever les contraintes.

\section{CONFLIT D'INTERETS}

Les auteurs déclarent qu'il n'y a aucun conflit d'intérêts.

\section{CONTRIBUTIONS DES AUTEURS}

Ce travail a été réalisé en collaboration entre tous les auteurs. ACE, HP, AP, OF ont conçu l'étude, ont écrit le protocole et ont fourni une revue approfondie de la littérature et des implications politiques et économiques des résultats de l'étude. ACE, HP, OF, KH ont collecté les données. ACE a effectué les analyses statistiques et élaborer le premier draft du manuscrit. AP et DG ont supervisé l'ensemble du travail et examiné toutes les ébauches du manuscrit. Tous les auteurs ont lu et approuvé le manuscrit final.

\section{REMERCIEMENTS}

Les auteurs remercient le Programme d'Amélioration de la Productivité Agricole des Petits Exploitants (PAPAPE) financé par la Banque Islamique de Développement (BID) qui a supporté cette étude.

\section{REFERENCES}

Adégbola PY, Sodjinou E, Singbo A. 2002. Etude de la compétitivité de la riziculture béninoise. Actes du 4Rs. http://hubrural.org/IMG/pdf/etude_comp etitivite_riziculture_beninoise.pdf

Ahouandjinou MC, Adegbola PY, Yabi JA, Adekambi SA. 2010. Adoption et impact socio-économique de la semimécanisation du procédé de transformation des amandes de karité en beurre au nord-Bénin. Contributed Paper presented at the Joint 3rd African Association of Agricultural Economists (AAAE) and 48th Agricultural Economists Association of South Africa (AEASA) Conference. Cape Town. South Africa.

Allogni WN, Coulibaly ON, Honlonkou AN. 2004. Impact des nouvelles technologies de la culture de niébé sur le revenu et les dépenses des ménages agricoles au Bénin. Bulletin de la Recherche Agronomique du Bénin, Numéro 44.

Appunu C, Sen D, Singh MK, Dhar B. 2008. Variation in symbiotic performance of Bradyrhizobium japonicum strains and Soybean cultivars under field condition. Central European Agriculture Journal, 9: $185-190 . \quad$ DOI : https://jcea.agr.hr/articles/561. 
Atidegla CS, Sintondji LO, Hounkpe J, Kpadonou E. 2017. Effets du labour mécanisé successif sur le statut nutritif du sol et le rendement du Riz pluvial dans la commune d'Abomey Calavi (Sud Bénin). European Scientific Journal, 13(30): $341 . \quad$ DOI: 10.19044/esj.2017.v13n30p341.

Balasubramanian V, Sie M, Hijmans RJ, Otsuka K. 2007. Increasing rice production in sub-saharan Africa: Challenges and opportunities. Advances in Agronomy, 94: 55$133 . \quad$ DOI: $10.1016 /$ S00652113(06)94002-4.

Balse M, Ferrier C, Girard P, Hvard M, Herbel D. 2015. Une expérience originale de mécanisation partagée en Afrique. Les coopératives d'utilisation de matériel agricole du bénin. http://agritrop.cirad.fr/575878.

Binswanger HP, Donovan G. 1987. La mécanisation agricole. Problèmes et options. Washington. Banque Mondiale., $95 \mathrm{p}$.

Christensen BHR. 2015. Analysis of ordinal data with cumilative link modelsestimation with the R-package ordinal. https://r-forge.r-

project.org/scm/viewvc.php/*checkout*/ $\mathrm{pkg} /$ ordinal/inst/doc/primer.pdf?revision $=66 \&$ root $=$ ordinal $\&$ pathrev $=69$.

Clarke L, Bishop C. 2002. Farm PowerPresent and Future Availability in Developing Countries. Invited Overview Paper Presented at the Special Session on Agricultural Engineering and International Development in the Third Millennium. ASAE Annual International Meeting/CIGR World Congress. Chicago. IL. USA.

FAO. 2016. La mécanisation agricole: un intrant essentiel pour les petits exploitants d'Afrique Subsaharienne. Gestion Intégrée des Cultures (Volume 23). $50 \mathrm{p}$. http://www.fao.org/documents/card/en/c/ cd9a50a3-c244-4217-81ff-bb7f03ff6557/

Fonteh FM. 2010. Agricultural Mechanization in Mali and Ghana: strategies, experiences and lessons for sustained impacts. FAO, Working document 8 . http://www.fao.org/fileadmin/user_uploa d/ags/publications/K7325e.pdf.

Grimonprez Q, Jacques J. 2016. Rankcluster: Model-Based Clustering for Multivariate Partial, Ranking Data. R package version 0.94. https://CRAN.Rproject.org/package $=$ Rankcluster.

Jaliya MM, Falaki AM, Mahmud M, Sani YA. 2008. Effect of sowing date and npk fertilizer rate on yield and yield components of quality protein maize (zea mays L.). ARPN Journal of Agricultural and Biological Science, 3(2):23-29.

Li-Juan Q, Ru-Zhen C. 2010. The origin and history of Soybean. In The Soybean: Botany, Production and Uses, Singh G (ed). CABI: Oxford.

Mrema CG, Baker D, Kahan D. 2008. Agricultural Mechanization in subsaharan Africa: time for a new look. FAO. Occasional paper 22. ftp://ftp.fao.org/docrep/fao/011/i0219e/i0 219e00.pdf

R. Core Team. 2017. $\mathrm{R}$ version 3.4.0.The $\mathrm{R}$ Foundation for Statistical Computing.

Side SC, Havard M. 2015. Développer durablement la mécanisation pour améliorer la productivité de l'agriculture familiale en Afrique subsaharienne. Int. J. Adv. Stud. Res. Africa. 6 (1\&2): 34-43.

Soulé BG, Yerima B, Soglo A, Vidégla E. 2008. Rapport diagnostique du secteur agricole du Bénin : Synthèse réalisée dans le cadre de la formulation du PNIA. ECOWAP/PDDAA., 124p.

Vall E, Dugué P, Blanchard M. 2006. Le tissage des relations agriculture-élevage au fil du coton, 1990-2005. Cahiers Agriculture, 15(1): 72-79. DOI : http://revues.cirad.fr/index.php/cahiersagricultures/article/view/30562.

Yuan Z. 2016. La mécanisation de l'agriculture en Afrique de l'Ouest. Fondation Syngenta pour l'agriculture durable.

https://www.syngentafoundation.org/site s/g/files/zhg576/f/la_mecanisation_de_la griculture_en_afrique_de_louest__yuan_zhou.pdf. 